\title{
Decomposability and Relative Invariance: the Structural Approach to Network Complexity and Resilience
}

\author{
Roberto Scazzieri ${ }^{1,2}$ (D)
}

Accepted: 13 January 2021/Published online: 16 June 2021

(C) The Author(s) 2021

\begin{abstract}
Economic complexity highlights the relationship between interdependence (a positional characteristic of elements belonging to a given network or structure) and connectivity (a functional characteristic of elements belonging to a given field of interaction). Positional interdependence (as the one between pieces in a jigsaw puzzle) is central to studies investigating the architecture of a complex system (Simon) while connectivity is central to the analysis of responsiveness patterns in social networks and strategic action fields. This paper discusses the fundamentals of a structural approach to economic and spatial complexity by highlighting the hierarchical arrangement of network elements as a distinctive feature of system identity. The positional distribution of network elements is a fundamental characteristic of complex networks and a central condition constraining the dynamics of those networks through the principle of relative structural invariance. The paper investigates the role of this principle by connecting it with the aggregation criterion followed in assigning network elements to specific subsystems. The type of aggregation is essential in determining the resilience properties of the network with respect to specific dynamic impulses. The paper concludes highlighting the need to combine the investigation of positional interdependence with the analysis of connectivity since positional interdependence is fundamental in determining which patterns of connection are more likely to arise (and which ones are excluded), due to the role of alternative properties of relative invariance constraining the feasible transformations in the positions of network elements.
\end{abstract}

Keywords Network complexity · Decomposability $\cdot$ Relative structural invariance · Aggregation criteria $\cdot$ Resilience and dynamic trajectories

I am grateful to two anonymous referees for their enlightening comments and suggestions. The usual caveat applies.

Roberto Scazzieri

rs292@cam.ac.uk; segreteria@lincei.it

Extended author information available on the last page of the article 


\section{Introduction: Positional Versus Functional Interdependence}

Any given network as a structure of mutually related components rests on the fundamental distinction between interdependence (a positional characteristic of elements belonging to that network or structure, such as the relationship between pieces in a jigsaw puzzle) and connectivity (a functional characteristic of elements involved in a domain of mutual responses, such as the relationship between actors in an action field). ${ }^{1}$ Elements belonging to a given economic and spatial network may be 'positionally dependent' on one another (in the sense that displacement of one element involves displacement of one or more elements within the same topology of possible positions) even in the absence of direct interaction between elements within that structure. On the other hand, elements belonging to a given field of interaction may be mutually responsive to one another even in the absence of positional changes within that field. Positional interdependence is central to studies highlighting what has been called the 'architecture of complexity' (Simon 1962), while functional interdependence is central to studies investigating connectivity in social networks and strategic action fields (Goyal 2007; Fligstein and McAdam 2011, 2012). This paper argues that a structural approach to economic complexity based on the distinction between positions and interactions could account for features of resilience that may otherwise remain unexplained. From a more general point of view, this paper aims to contribute to the interdisciplinary dialogue between the positional (spatially embedded) approaches to network analysis and the approaches addressing the complex dynamics triggered by nonlinearities in the interacting components of the system under investigation (Ducruet and Beauguitte 2014; Tsiotas and Polyzos 2018).

Section 2 outlines the fundamentals of the structural approach to economic and spatial complexity by highlighting the role of the hierarchical arrangement of system elements as a central feature of system identity. This section considers the positional distribution of system elements as a fundamental characteristic of complex systems and a most important factor in determining the dynamics of complex systems through a principle of relative structural invariance. This principle states that not all system elements can change at the same time and/or at the same speed.

Section 3 carries this argument into dynamic analysis by connecting the working of relative structural invariance with the aggregation criterion followed in assigning system elements to specific subsystems (such as clusters of firms, industries, or vertically integrated productive sectors). This section highlights that different aggregation criteria may be associated with different bottlenecks and/or windows of opportunity due to the greater salience of certain invariances relative to others. As a result,

\footnotetext{
${ }^{1}$ The distinction between positional and functional characteristics is central to the argument of this paper and involves the view of connectivity as '[t] he characteristic, or order, or degree, of being connected', as in A. R. Forsyth's Theory of Functions of a Complex Variable: '[f]or a simply connected surface [...] the connectivity is unity' (Forsyth 1893, §160).

(See https://www-oecom.ezp.lib.cam.ac.uk/view/Entry/39340?redirectedFrom=Connectivity\#eid; accessed 16.10. 2020). This definition highlights features of an actual relational state and is therefore distinct from the view of connectivity as the 'capacity for the interconnection of platforms, systems, and applications', which is a virtual property commonly addressed in computer science (https://www.lexico.com/definition/ connectivity; accessed 16.10.2020, added emphasis).
} 
important features of the dynamics of economic and spatial networks depend on which type of aggregation is empirically and operationally more important in specific contexts.

Section 4 builds on the analysis of the previous section and examines the relationship between the aggregation criterion adopted in assigning network elements to specific subsystems and the resilience properties of the economic system with respect to specific dynamic impulses. This section argues that a structural approach to economic and spatial complexity may account for features of vulnerability and resilience that may otherwise remain unnoticed and unexplained.

Section 5 brings the paper to close by highlighting the need to combine the analysis of connectivity with the investigation of positional interdependence seeing that the configuration of interdependence may be central in determining which patterns of connectivity are more likely to arise (and which ones are excluded) due to the invariance of certain relative positions of network elements. This section also highlights that the distribution of relative invariances at a given point of time and over time, by determining which changes of relative positions are feasible and which ones are not, is an important factor in explaining the routes taken by the structural dynamics of economic and spatial systems as they steer between different and sometime opposed patterns of resilience.

\section{Architecture of Complexity, Internal Hierarchies, Structural Invariance}

\subsection{Relative Positions and Invariance}

Jean Piaget argued that 'the character of structured wholes depends on their laws of composition', so that 'these laws must of their very nature be structuring', and thus must give rise to a 'constant duality or bipolarity, of always being simultaneously structuring and structured' (Piaget 1971 [1968], p. 10; author's emphasis). Laws of composition provide the analytical framework for the study of the way in which the economic system may react to internal or external triggers of change. The 'architecture of complexity' (Simon 1962, 1976) is to a large extent coincident with the law of composition characterizing any given pattern of interdependencies and its internal hierarchy. Architecture of complexity as defined above is central in economic and spatial networks due to the fundamental importance in those networks of relative positions and relative distances. ${ }^{2}$ The hierarchy between different system elements (subsystems) is at the root of the criterion of relative structural invariance. By this criterion, different components of an economic and spatial network may change at different speeds, so that, under certain conditions, some of those components are invariant

\footnotetext{
${ }^{2}$ As Reggiani notes: 'spatial networks [are] networks where space - in the form of distance friction and/or transportation/communication costs/utilities - assumes a fundamental role' (Reggiani 2014, p. 812, added emphasis). Distance itself can be approached in different ways depending on whether it is measured as Euclidean distance or as distance in a non-Euclidean space, such as a proximity space à la Efremovič (Naimpally and Warrack 1970). In the latter case, distance is associated with a vector of characteristics, and relative distances will vary depending on which characteristic (or set of characteristics) is privileged in measurement.
} 
while other components may change at variable speeds. For example, certain industries or regions may be more responsive than others to technical change or to scarcity bottlenecks, and the diffusion of certain innovations may follow a technically constrained path depending on the 'order of motion' between the different elements of the production process (Dahmén 1970, 1984). Relative structural invariance reflects the fact that, in general, any economic and spatial network 'subject to an impulse or force is allowed to change its original state by following an adjustment path that belongs to a limited set of feasible transformations [ ...] In this way, the impulse from which the original state $[\ldots]$ is modified may be purely exogenous but the actual process of transformation can be explained in terms of the "dynamics" characteristics of the existing structure (that is in terms of the specific paths of feasible transformations that are compatible with its description)' (Landesmann and Scazzieri 1990, p. 96). At the origin of the relative invariances in economic and spatial networks is the resilience of certain network elements relative to other elements (Landesmann and Scazzieri 1996), or the permanence of certain relationships between elements relative to other relationships (Dagum 1969). An illustration of the criterion of relative structural invariance is provided by the network of intermediate product flows in an industrial system characterised by advanced division of labour and given technology (Leontief 1991 [1928]), 1941; Pasinetti 1977]; see also Ames and Rosenberg 1965; Yang and Ng 1993).

\subsection{Relative Invariance and Resilience}

The case of intermediate product flows mentioned at the end of the previous subsection may be associated with the resilience of network elements relative to a specific time

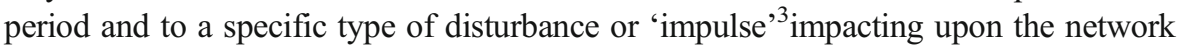
under consideration. A standard representation of intermediate product flows in a system of interdependent production activities is the one adopted in Wassily Leontief's interindustry analysis (Leontief 1941; Leontief et al. 1953). In interindustry analysis, the structure of intermediate product flows is usually described in terms of a matrix $\boldsymbol{A}$ of 'technical coefficients' $a_{i j}$ denoting the quantity of input $i$ that enters the production of each unit of good $j$ (see, for example, Pasinetti 1977) ${ }^{4}$ :

$$
\boldsymbol{A}=\begin{array}{cccc}
a_{11} & a_{12} & \ldots & a_{1 m} \\
a_{21} & a_{22} & \ldots & a_{2 m} \\
\ldots & \ldots & & \ldots \\
a_{m 1} & a_{m 2} & \ldots & a_{m m}
\end{array}
$$

The above configuration of interindustry product flows suggests a network structure (or, in the appropriate context, a spatial structure) in which each industry is connected by product flows with any other industry of the given system. This suggests for the above configuration of product flows the structure of a fully connected graph $\mathbf{G}(I, E)$

\footnotetext{
3 The concept of 'impulse' as a positive or negative shock in economic dynamics was introduced by John Hicks in his Nobel lecture (Hicks 1977 [1973], p. 15)

${ }^{4}$ Square matrix $\boldsymbol{A}$ represents a configuration of production interdependencies in which each industry acquires and delivers product flows from/to any other industry of the system. It is as such the prototypical case of a circular economy. Subsection 3.2 discusses an analytical device by which rectangular matrices of interindustry flows can be reduced to the square matrix case.
} 
(where the nodes $I$ are the industries and the edges $E$ are the interindustry product flows connecting industries with one another). ${ }^{5}$ An example would be a 3 -industry fully connected network as below:

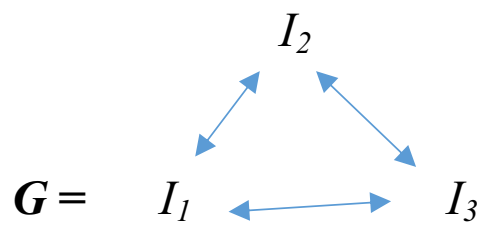

The resilience of a production network such as the one represented in graph $\mathbf{G}(I, E)$ can be expressed using the following notation: $R_{i j}\left(\left\{I_{i}\right\}, D_{j} t^{*}\right)$ where $R_{i j}$ is the resilience of network element $I_{i}$ subject to the disturbance or impulse $D_{j}$ in time period $t^{*}$ (Landesmann and Scazzieri 1996, p. 340). $R_{i j}\left(\left\{I_{i}\right\}, D_{j}, t^{*}\right.$,) may be either 1 or 0 depending on whether network element $I_{i}$ is resilient or not under impulse $D_{j}$ in time period $t^{*}$. This concept of resilience is closer to the notion of 'ecological resilience' than to the notion of 'engineering resilience', as the former notion highlights the ability of a system, or system component, to withstand a shock rather than the ability of that system, or system component, to return to a previous equilibrium state after being displaced from it (Holling 1973; Modica and Reggiani 2015). In our case, resilience encompasses both the possibility that a given network element returns to a previous equilibrium state after a shock or 'impulse' and the possibility that a network element will move to a different position within the same 'buffer zone' of feasible transformations (that is, within the zone compatible with the persistence of the set of interdependences characterizing the industrial system under consideration).

\subsection{Aggregation, Disaggregation and Resilience}

The architecture of economic and spatial networks may be represented through alternative ways of aggregating economic activities into subsystems. A classic distinction in economic theory is the one between decomposition of a given economic network by industries or by vertically integrated sectors (Pasinetti 1980 [1973]). Each decomposition criterion is associated with different proportionality conditions regulating the relationship between different types of motion within the system. Alternative patterns of decomposition make certain patterns of interdependence feasible and others unfeasible. In the light of this property, the relationship between modes of positional interdependence and connectivity emerges as a central element in the explanation of relative invariance and resilience. For example, a pattern of interdependence based on network decomposition by industry highlights proportionality requirements that have to be satisfied by industries delivering intermediate inputs to one another, thus making the economic system vulnerable to disruptions affecting the provision of intermediate inputs at any given time, while a pattern of interdependence based on network

\footnotetext{
5 The relationship between interindustry analysis and the network view of the economic system is highlighted in a paper by Wassily Leontief originally published in 1928 (Leontief 1928; see its partial English translation in Leontief 1991 [1928]). Leontief's references to François Quesnay’s Tableau économique (Quesnay 1972 [1759]) are further illustration of the network foundations of interindustry (input-output) analysis (Leontief 1941).
} 
decomposition by vertically integrated sectors highlights a constraint due to complementarities along sequentially arranged stages of production, thus making the economic system vulnerable to disruptions affecting the delivery of materials-in-process along particular chains of production.

The above duality highlights the different role of resilience in the two cases. Let $s_{i j}$ $\left(t^{*}\right)$ be the stock of intermediate input $i$ available to a given industry in time period $t^{*}$ when the network is subject to impulse $D j$ and let $u_{i j}\left(t^{*}\right)$ be the quantity of intermediate input $i$ required in the same industry at time period $t^{*}$ when the network is subject to impulse $j$ (Landesmann and Scazzieri 1996, p. 325). The relationship between $u_{i j}\left(t^{*}\right)$ and $s_{i j}\left(t^{*}\right)$ is critical in determining whether network element $I_{i}$ (the industry under consideration) will be resilient or not under impulse $D_{j}$ in time period $t^{*}$. As a general condition, we have $I_{i}=1$ ( $I_{i}$ resilient) if $u_{i j}\left(t^{*}\right) \leq s_{i j}\left(t^{*}\right)$ and $I_{i}=0$ ( $I_{i}$ non resilient) if $u_{i j}$ $\left(t^{*}\right)>s_{i j}\left(t^{*}\right){ }^{6}$ However, the relationship between $u_{i j}\left(t^{*}\right)$ and $s_{i j}\left(t^{*}\right)$ may be different depending on the type of decomposition adopted. With decomposition by industry, we may have $u_{i j}\left(t^{*}\right) \leq s_{i j}\left(t^{*}\right)$ provided the direct requirements of intermediate input $i$ by a given industry stay below the corresponding available stock $s_{i j}\left(t^{*}\right)$. On the other hand, decomposition by vertically integrated sectors highlights direct and indirect requirements for intermediate input $i$, and may therefore reveal a situation in which the quantity of that intermediate input which would be required in time period $t^{*}$ in some of the industries contributing to a particular supply chain $I_{i}$ following impulse $D_{j}$ would be greater than the quantity available $\left(u_{i j}\left(t^{*}\right) \leq s_{i j}\left(t^{*}\right)\right)$. In this case, network element $I_{i}$ (the vertically integrated sector, or supply chain, under consideration) will not be resilient even if some or most of its contributing industries are.

\subsection{Stable Intermediate Forms and near-Decomposability}

Herbert Simon argued that 'complexity frequently takes the form of hierarchy', and that 'hierarchic systems have some common properties that are independent of their specific content' (Simon 1962, p. 468). In Simon's view, complex systems are generally 'composed of interrelated subsystems, each of the latter being, in turn, hierarchic in structure until we reach some lowest level of elementary subsystem' (Simon 1962, p. 468). The decomposition of the economic system into relatively independent subsystems has important consequences for the way in which the system reacts to internal or external impulses, as well as for the way in which the system may or may not undergo a transformation of the relationship between its components (that is, on the structural dynamics that system is likely to follow). Simon's approach to system structure is a fundamental building block in the analysis of relative structural invariance (see section 1 for a discussion of this concept). In Simon's view, the two fundamental structural features influencing relative invariances and therefore system dynamics are: (i) intermediate stable forms, and (ii) near decomposability.

Intermediate stable forms allow a complex system to evolve by changing the relationship between components in a non-disruptive way. In Simon's words: '[ $t$ ]he time required for the evolution of a complex form from simple elements depends

\footnotetext{
${ }^{6}$ The above condition highlights a link between the resilience of an industrial network and the redundancy of (at least) some of the flows of intermediate products in that network (see also Bruneau et al. 2003; Reggiani 2013).
} 
critically on the numbers and distribution of potential intermediate stable forms. In particular, if there exists a hierarchy of potential stable "subassemblies," with about the same span, $s$, at each level of the hierarchy, then the time required for a subassembly can be expected to be about the same at each level' (Simon 1962, p. 471). As a result, 'the existence of stable intermediate forms exercises a powerful effect on the evolution of complex forms that may be likened to the dramatic effect of catalysts upon reaction rates' (Simon 1962, p. 472) and 'complex systems will evolve from simple systems much more rapidly if there are stable intermediate forms than if there are not. The resulting complex forms in the former case will be hierarchic' (Simon 1962, p. 473).

Strictly associated with the system property giving rise to intermediate stable forms is near decomposability. In this case, "we can distinguish between the interactions among subsystems, on one hand, and the interactions within subsystems -i.e., among the parts of those subsystems-on the other' (Simon 1962, p. 473, author's emphasis). A near-decomposable system exhibits a hierarchy between types of motion within the system such that: '(a) [...] the short- run behavior of each of the component subsystems is approximately independent of the short-run behavior of the other components; (b) in the long run, the behavior of any one of the components depends in only an aggregate way on the behavior of the other components' (Simon 1962, p. 474).

Hierarchy is at the root of both structural dynamics, defined as change in the relationship between system components independently of which impulse originates the transformation of system structure, and of reactive dynamics, defined as the system's response to specific impulses. Intermediate stable forms and near decomposability are two facets of the relative structural invariance characterizing the motion of hierarchically ordered systems. The relative persistence of 'sub-assemblies' allows changes to take root in the system through a piecemeal process that allows structural dynamics to unfold in spite of interruptions in the working of the dynamic impulses triggering transformation (such as a temporary slowdown of technical progress or a temporary fall of per capita incomes). Similarly, the short-run mutual independence of the dynamics of subsystems in a nearly decomposable system allows dynamic impulses (such as a sudden fall of effective demand or a sudden dislocation in the "channels of trade') ${ }^{7}$ to be temporarily contained within specific subsystems and therefore not to disrupt the fundamental (long-term) evolution of the economic system under consideration.

In short, both intermediate stable forms and near-decomposability work to the same result by making it possible for the economy to undergo the transformations associated with a specific dynamic trajectory despite the temporary interruption of dynamic impulses.

\subsection{Relative Invariance as Dynamic Principle}

The joint working of intermediate stable forms and near decomposability highlights the role of relative structural invariance as the fundamental dynamic principle governing

\footnotetext{
${ }^{7}$ David Ricardo illustrates this type of dynamic shocks by considering 'temporary reverses and contingencies, produced by the removal of capital from one employment to another' (Ricardo 1951 [1817], Chapter XIX 'On Sudden Changes in the Channels of Trade', p. 263).
} 
the motion of a hierarchically ordered economic and spatial network. Decomposability of the system into a collection of subsystems results from the application of principles of aggregation and disaggregation, which means that any given system may be associated with different collections of subsystems depending on which principles of aggregation/disaggregation are followed.

In turn, the decomposition of the system by a given principle of aggregation/ disaggregation may bring to light intermediate stable forms different from the intermediate stable forms associated with a different principle of aggregation. For instance, decomposition of the economic system by industries delivering a homogeneous product highlights the possibility of intermediate stable forms (industries) different from the vertically integrated sectors (chains of production) delivering final consumption goods by means of a sequence of different semi-finished products leading up to those goods.

We may conjecture that the relative salience of one or another type of intermediate stable forms would lead to different trajectories of structural change as each collection of intermediate stable forms is likely to be more receptive to certain types of impulses than to others. For instance, decomposition by interdependent industries is likely to make the economic and spatial network more responsive to changes in a generalpurpose technology (a technology affecting by definition all industries in the system); on the other hand, decomposition by vertically integrated sectors is likely to make the network more responsive to 'specialized' types of technical change affecting different sequences of fabrication stages leading to different final goods.

We may also conjecture that different criteria of decomposition of the economic system would be associated with different distribution patterns of weak and strong ties across economic activities. For instance, decomposition by industries highlights strong ties between the core industries delivering intermediate products to one another (basic industries) and weak ties between that core and all other industries; while decomposition by vertically integrated sectors highlights strong ties between the fabrication stages belonging to any given sector and weak ties between fabrication stages belonging to different sectors (different chains of production). ${ }^{8}$

As we shall see in the following section, dominance of one decomposition over others may lead to important differences in the dynamic trajectory followed by the economic system as it may lead to different patterns of responsiveness to dynamic impulses, and to different patterns or resilience to major or minor disturbances. In particular, alternative decomposition criteria may have important consequences for what concerns the relationship between structural dynamics and reactive dynamics, and therefore for the structural transformations associated with the reaction of a resilient socio-economic system to external or internal disturbances. In fact, resilience may often require structural change, so that resilience dynamics and structural dynamics may often go hand in hand. This means that resilience dynamics is often associated with the system's capability to modify its own structure by taking in new components and /or by switching to a different disposition of existing components. However, the system's ability to activate one or another trajectory of

\footnotetext{
${ }^{8}$ In production networks, strong ties characterize the connections between processes, or supply chains, that are mutually necessary for their respective functioning. On the other hand, weak ties characterize the connections between processes, or supply chains, that can be substituted with connections with other processes or supply chains without disrupting the functioning of the whole system.
} 
resilience dynamic critically depends on dominance of one system decomposition over another.

In this light, structural analysis complements the view of the economy as an adaptive, evolving system subject to nonlinear phenomena, which is dominant in the self-organization and structural emergence literature (Ross Ashby 2004 [1962], ${ }^{9}$ Baas 1994) by highlighting the constraining and orienting role of structures and of the associated patterns of relative invariances along transformation trajectories (Landesmann and Scazzieri 1990; Arthur et al. 1991; Cardinale and Scazzieri 2019; Scazzieri 2021). For instance, decomposition by industries may require restructuring according to the hierarchical arrangement of industries, which in turn suggest restructuring starting with the system's core activities (basic industries). On the other hand, decomposition by vertically integrated sectors may allow the system to undergo restructuring by rearranging specific supply chains, as well as the proportions between supply chains, without undergoing a radical restructuring of its basic industries.

\section{Circular Vs. Vertical Network Structures and Dynamic Trajectories}

\subsection{Alternative Network Decomposition Criteria}

The relative invariance (resilience) of certain elements of network structure with respect to other elements of the same structure is fundamental in determining the system's dynamic behaviour, both in the sense of the evolution of interdependencies between system components (system's structural dynamics) and in the sense of the system's responsiveness to disturbances (system's stability dynamics). The aim of this section is to illustrate the different working of relative structural invariance depending on which decomposition criterion is dominant. To that purpose, this section considers two alternative representations of the network structure of the economy: the circular representation by industries delivering intermediate products to one another and the representation by vertically integrated sectors connecting one fabrication stage to another along production sequences leading to different final consumption or final investment goods. As we shall see, structural dynamics and stability dynamics may be different depending on whether the dominant decomposition criterion for network structure is of the circular or of the vertical type.

\footnotetext{
${ }^{9}$ N.A. Baas views self-organization has a property of systems displaying new characteristics ('emergent structures') when switching from lower to higher levels of aggregation (Baas 1994). W. Ross Ashby's discussion of organizational dynamics in an abstract 'machine' makes changes of state dependent on identification of the set of states that a given organization can be in: "[t]he "organization" must [...] be identified with $f$, the mapping of $\boldsymbol{S}$ into $\boldsymbol{S}$ that the basic drive of the machine (whatever force it may be) imposes. Now the logical relation here is that $f$ determines the changes of $\boldsymbol{S}: f$ is defined as the set of couples $\left(s_{i}, s_{j}\right)$ such that the internal drive of the system will force state $s_{i}$ to change to $s_{j}^{\prime}$ (Ross Ashby 2004 [1962], pp. 115-116). Ross Ashby's view is compatible with the possibility that structural dynamics within $\boldsymbol{S}$ may trigger certain changes from one state to another while making other changes impossible. This possibility provides a link between structural emergence and the role of existing (historical) structures as conditions for structural change.
} 
In the circular case, the network structure of the economy may be represented by a matrix describing the flows of intermediate products linking one industry to another. Matrix $\boldsymbol{A}$ in section 2.2 above describes intermediate product flows in a circular $m$-good economy (each element $a_{i j}$ denotes the quantity of good $i$ needed to produce each unity of $\operatorname{good} j)^{10}$ :

$$
\boldsymbol{A}=\begin{array}{llll}
a_{11} & a_{12} & \ldots & a_{1 m} \\
a_{21} & a_{22} & \ldots & a_{2 m} \\
\ldots & \ldots & & \ldots \\
a_{m 1} & a_{m 2} & \ldots & a_{m m}
\end{array}
$$

In the vertical case, the network structure of the economy may be represented by a set of $m$ vertically integrated sectors describing $m$ lines of fabrication stages that coincide with the $m$ final goods produced in the economy. Matrix $\boldsymbol{H} \equiv\left[\mathbf{h}_{i}\right](i=1, \ldots, m)$ below represents intermediate product flows rearranged by vertically integrated sectors, in which each vector $\mathbf{h}_{i}$ 'expresses in a consolidated way the series of heterogeneous physical quantities of commodities $1,2, \ldots, m$, which are directly and indirectly required as stock, in the whole economic system, in order to obtain one physical unit of commodity $i$ as a final $\operatorname{good}(i=1,2, \ldots, m)^{\prime}$ (Pasinetti 1980 [1973], pp. 20-21):

$$
\mathbf{H}=\left[\mathbf{h}_{1}, \mathbf{h}_{2}, \ldots, \mathbf{h}_{\mathrm{m}}\right]
$$

Matrices $\boldsymbol{A}$ and $\boldsymbol{H}$ represent the same network structure in two different ways.

Matrix $\boldsymbol{A}$ highlights the circular flow characterizing the mutual deliveries of intermediate products across industries, while matrix $\boldsymbol{H}$ describes vertical 'one-way' flows characterizing the deliveries of work-in-process goods from one fabrication stage to another along the productive sequences delivering different final goods. The two matrices reflect different approaches to network structure and highlight different invariances within that structure. Matrix $\boldsymbol{A}$ highlights the organization of productive activities by industries (in principle, each industry produces a homogeneous good by a single technique). In this case, industries themselves can be stable intermediate forms relatively persistent over time, while the distribution of weak and strong ties would broadly follow the distinctions between different industries. In other words, the principle of relative structural invariance is industry-based. As a result, the economy would behave as a near-decomposable system of industries. This means that in the short-term dynamic impulses (of the positive or negative type) would influence the system in a distinct way depending on which industries are considered, while the subsequent filterdown effect from the macro-level to individual subsystems would follow the route of industries rather than the route of vertically integrated sectors (chains of production).

Differently from matrix $\boldsymbol{A}$, matrix $\boldsymbol{H}$ calls attention to the organization of productive activities by $m$ different vertically integrated sectors (in principle, collections of

\footnotetext{
${ }^{10}$ Matrix $\mathbf{A}$ is a square matrix describing a situation in which there is one-to-one correspondence between production processes and goods produced. The cases in which certain commodities are produced by a plurality of processes, or certain processes produce a plurality of commodities, can be reduced to the square matrix case by means of the 'splitting coefficients' method proposed by A. Quadrio Curzio $(1967,1996)$ and discussed below.
} 
sequentially connected fabrication stages leading to the production of homogeneous final goods of the consumption or investment type). In this case, vertically integrated sectors may be considered as relatively persistent intermediate stable forms, while links between activities will be strong within each vertical sector and weak across different vertical sectors. In this case, relative structural invariance follows the decomposition of the network into semi-independent chains of production. The economy would behave as a near-decomposable system of vertically integrated sectors, so that short-term dynamic impulses affecting a particular sector would not be immediately transmitted to the rest of the economy, and the subsequent filter-down effect from the macro-level to individual system components would follow the route of sequentially connected stages of semi-finished products rather than the route of industries producing a homogeneous final product.

\subsection{One Good, Many Techniques; One Technique, Many Goods}

The distinction between decomposition criteria may be further explored by dropping the assumption of one-to-one correspondence between goods and techniques, that is, by allowing techniques delivering multiple products and products made by multiple techniques. In this case, matrix $\boldsymbol{A}$ and matrix $\boldsymbol{H}$ need to be substantially transformed. For example, if one good (say, good 1) is produced by two different techniques I and II, matrix $\boldsymbol{A}$ may be written as follows:

$$
\boldsymbol{A}=\begin{array}{rrrrr}
a_{11}(\mathrm{I}) & a_{11}(\mathrm{II}) & a_{12} & \ldots & a_{1 \mathrm{~m}} \\
a_{21}(\mathrm{I}) & a_{21}(\mathrm{II}) & a_{22} & \ldots & a_{2 \mathrm{~m}} \\
\ldots & \ldots & & & \\
a_{m 1}(\mathrm{I}) & a_{m 1}(\mathrm{II}) & a_{m 2} & \ldots & a_{m m}
\end{array}
$$

This approach to the circular decomposition of network structure raises a new set of questions concerning relative structural invariance and its realisation through emergence of intermediate stable forms and near decomposability. In fact, there will be industries that are 'split' into two (or more) processes depending on the number of techniques simultaneously in use (as is the case with industry 1 in matrix $\boldsymbol{A}$ above). A relevant issue in this connection is the assignment of weights to the different processes delivering the same good (in our example good 1) by using differing techniques (that is, different vectors of technical coefficients $a_{i j}$ ). The introduction of 'splitting coefficients' may be of use to this purpose: 'The technology of the economic system can now be represented as with two "jointed techniques" or with a "global technology" $\boldsymbol{A}_{\alpha}$ (I, II). The compact notation $\boldsymbol{A}_{\alpha}$ (I, II) indicates that it is possible to include [...] in this new type of technological matrix two processes that produce [good 1], and that this matrix varies with changes in the "splitting coefficients" $\alpha_{\mathrm{s}}$ (these are the coefficients used to "split", in proportions to be determined, the known technical coefficients) that connect [good 1] as input to all other $m$ processes of the economy' (Quadrio Curzio 1986, p. 324; see also Quadrio Curzio and Pellizzari 1999, pp. 52-53).

Global technology $\boldsymbol{A}_{\alpha}$ (I, II) describes a production network in which 'two processes', such as al (I) and al (II), 'are now jointed' in the production of commodity 1 'which is required by the whole economic system' (Quadrio Curzio 1996, p. 112). In 
this case, the " "splitting or split supply coefficients" $(\alpha)$ identify the extent to which processes [I and II], respectively, supply [commodity 1] to every other process of the economic system' (Quadrio Curzio 1996, p. 112). This network structure is represented in the technology matrix below:

$$
\begin{array}{cllll}
a_{11}(I) & 0 & \alpha_{12}(I) & \cdots & \alpha_{1, m}(I) \\
0 & a_{11}(I I) & \alpha_{12}(I I) & \cdots & \alpha_{1, m}(I I) \\
a_{21}(I) & a_{21}(I I) & a_{22} & \cdots & a_{2, m} \\
\cdots & \ldots & \cdots & \cdots & \cdots \\
a_{m, 1(I)} & a_{m, 1}(I I) & a_{m 2} & \cdots & a_{m m}
\end{array}
$$

In short, the introduction of splitting coefficients allows the decomposition of network structure by industries to be compatible with changing weights between the different processes delivering the same good along a dynamic trajectory, as it may happen with industries requiring multiple techniques due to resource bottlenecks, or with industries characterized by coexistence of old and new techniques. As to near decomposability, the emergence of 'split industries' raises the issue of what would be the privileged transmission channel of dynamic impulses (say, whether it would be technique I or technique II in the case above). This issue is of interest seeing that different impulses are likely to be associated with different transmission channels. For example, a resource bottleneck affecting the maximum level of activation for technique I is likely to induce a change of splitting coefficients that goes from higher to lower weights for the 'most profitable' technique I and from lower to higher weights for the 'least profitable' technique II. This means that the process by which the 'weights' of techniques I and II are changed may be accelerated or, vice versa, slowed down depending on changing resource availability.

On the other hand, the introduction of a technical innovation requiring a minimum process scale is likely to induce a process of changing splitting coefficients that goes from lower to higher weights for technique II (the 'most profitable' technique) and from higher to lower weights for technique I (the 'least profitable' technique). This means that the process of changing weights may be accelerated or, respectively, slowed down depending on the speed at which minimum scale thresholds can be overcome. Splitting coefficients are useful in representing the structural dynamics of a network of industries as they highlight the changing weights of different techniques within one or more industries. However, the specific character of a dynamic impulse may fundamentally alter the transmission mechanism associated with changes of splitting coefficients. In the resource bottlenecks case, higher output levels of good 1 are associated with higher weights for the 'least profitable' technique I and lower weights for the 'most profitable' technique II (decreasing returns trajectory), while in the minimum- scale (process indivisibility) case lower output levels of good 1 are associated with lower weights for the 'most profitable' technique II and higher weights for the 'least profitable' technique I (increasing returns trajectory) (see also Scazzieri 1993, 2014).

In the case of matrix $\boldsymbol{H}$ (decomposition by vertically integrated sectors), dropping the one-to-one correspondence between produced goods and production techniques requires the acknowledgement that different vectors of commodity stocks may be required in the economic system to produce one unit of any particular final good $i$ 
$(i=1,2, \ldots, m)$. For example, if good 1 is produced by two different techniques I and II, matrix $\boldsymbol{H}$ needs to be rewritten as follows:

$$
\mathbf{H}=\left[\mathbf{h}_{1}(\mathrm{I}), \mathbf{h}_{1}(\mathrm{II}), \mathbf{h}_{2}, \ldots, \mathbf{h}_{\mathrm{m}}\right]
$$

In this case, two vectors of commodity stocks (rather than a single vector) are required in the economic system to allow production of the required quantity of good 1. A relevant issue in this connection is the degree to which the commodity stocks that are directly or indirectly needed to produce good 1 , respectively with technique I or technique II, are mutually substitutable as intermediate inputs in the production of other goods in the economy. For example, it is likely that the commodity stocks required in the economic system to produce one unit of good $i(i=1,2, \ldots, m)$ with technique I (that is, vector $\mathbf{h}_{1}$ (I)) will be different from the commodity stocks required in the economic system to produce one unit of good $i(i=1,2, \ldots, m)$ with technique II (that is, vector $\mathbf{h}_{1}(\mathrm{II})$ ). In this case, a positive growth rate will be associated with the emergence of residuals, that is, with stocks of intermediate inputs that cannot be used in expanding production as long as the economy continues to substitute vertically integrated sector $\mathbf{h}_{1}$ (II) for vertically sector $\mathbf{h}_{1}$ (I) (Quadrio Curzio 1986, pp. 327-336). Decomposition of network structure by vertically integrated sectors provides an alternative way of expressing changing weights between different techniques delivering the same good along a structural change trajectory. This approach to the decomposition of economic structure has significant implications for the resilience (structural invariance) of network elements. There will be vertically integrated sectors that are 'split' into two (or more) subsectors depending on the number of techniques simultaneously in use (such as sector $\mathbf{h}_{1}$, which is split into sub-sectors $\mathbf{h}_{1}$ (I) and $\mathbf{h}_{1}$ (II) in matrix $\boldsymbol{H}$ above). This implies that the resilience of a particular vertically integrated sector, such as $\mathbf{h}_{1}$, is compatible with changes in the internal composition of the commodity stocks associated with that sector. This may happen, for example, when those commodity stocks reflect the joint use of multiple techniques due to resource bottlenecks, or to the coexistence of old and new techniques along a technical progress trajectory).

As to near decomposability, the emergence of vertically integrated sectors that are 'split' between two or more distinct subsystems raises the issue of which vector(s) of commodity stocks would be the privileged transmission channel(s) of dynamic impulses (for example, whether dynamic impulses would exert their effects through commodity stocks $\mathbf{h}_{1}$ (I) or through commodity stocks $\mathbf{h}_{1}$ (II) in matrix $\mathbf{H}$ above).

\subsection{Systems of Industries Vs. Systems of Vertically Integrated Sectors}

In conclusion, alternative modes of aggregation/decomposition may trigger alternative dynamic trajectories. The decomposition of an economic and spatial network in terms of a system of industries highlights the mutual dependencies connecting industries to one another into a potentially coherent and 'self-replacing' system. ${ }^{11}$ As we have seen, this mode of decomposition allows for changing weights between different techniques within one or more industries but does not highlight the emergence of radical

\footnotetext{
${ }^{11}$ A 'self-replacing' system is one in which 'each industry produces a quantity of commodity equal to the amount required of that commodity as a means of production by the entire system' (Bellino 2018, p. 850).
} 
discontinuities between different industries. The different industries are tied with one another through the reciprocal need for intermediate inputs and certain proportions between industries must be maintained for the viability condition to be met. On the other hand, decomposition of network structure by a system of vertically integrated sectors highlights the relative independence of the sequences of fabrication stages (chains of production) leading to the production of different final goods of the consumption or investment type. This mode of aggregation/decomposition presupposes the provision of required stocks of intermediate products $\mathbf{h}_{i}$ for each vertically integrated sector $i(i=1, \ldots, m)$ but does not presuppose that all vectors of intermediate commodity stocks $\mathbf{h}_{1}, \mathbf{h}_{2, \ldots}, \mathbf{h}_{\mathrm{m}}$ would be produced within the same system of industries.

In other words, the dominance of the vertical mode of aggregation/decomposition of economic activities into subsystems is compatible with the 'distribution' of the different $\mathbf{h}_{i} s$ vectors of commodity stocks between different systems of industries (for example, between different national economies). ${ }^{12}$ This implies that a given collection of vertically integrated sectors (which may coincide with an empirically identifiable economic system) may or may not be compatible with the viability requirements for the different systems of industries delivering the various $\mathbf{h}_{i} s$ commodity stocks. Dominance of vertical sectors is compatible with changes in sectoral proportions beyond the 'selfreplacing' constraint that would apply for each system of industries individually considered.

\section{Interdependence and Connectivity: Alternative Paths to Vulnerability and Resilience}

\subsection{Structure Vs. Connections: Implications for Vulnerability and Resilience}

The interdependence approach to network structure highlights the relative positions of network components vis à vis one another independently of whether those components are actively involved in mutual interaction. As a result, this approach considers network structure as a relatively invariant constellation of positions from which actual interactions between network elements arise. On the other hand, the connectivity approach highlights the mutual responsiveness of network elements to one another's actions independently of assumptions concerning the relative invariance of certain positions and the corresponding hierarchy of feasible responses. In the latter case, network structure appears as an emergent property reflecting evolving patterns of interaction

\footnotetext{
12 The vertical mode of decomposition may provide a heuristic for assigning specific vectors $\mathbf{h}_{i} s$ of intermediate commodity stocks to the system of industries to which they belong independently of the system of industries to which the corresponding final consumption or investment good belongs. In this way, vertical aggregation/decomposition may be considered a step towards inductive identification of systems of industries as production networks independently of institutional or political constraints. The Enlightenment economist Cesare Beccaria hinted at this possibility when he wrote that 'the political borders of a state are not always, or almost never, the same as its economic borders...[t] he land of a nation nourishes the industry of another, while the industry of the latter improves the fertility of the former: these two nations, even if divided in sovereignty and made reciprocally independent by their respective political laws, are in fact a single nation strictly united through physical laws and made mutually dependent by economic relations' (Beccaria 1971 [ms. circa 1769], p. 391, added emphasis).
} 
between network elements rather than relatively invariant characteristics of that structure. In other words, it is important to distinguish between 'interactions, that is, [...] actual social exchanges that actually take place' and 'structures' that 'cannot be reduced to the interactions between [say] two persons talking to each other' (Bourdieu 2012, p. 179).

The distinction between interdependence and connectivity has relevant implications for the way in which vulnerability and resilience are conceptualized in theory and addressed in policy. Vulnerability may be seen as a network's 'susceptibility to harm, powerlessness, and marginality' both from the physical and social point of view (Adger 2006 , p. 269), while resilience is often approached as a network's 'capacity to selforganise' by adapting 'to emerging circumstances' (Adger 2006, pp. 268-9). These definitions imply that a network can be vulnerable without being resilient and vice versa. However, in practice the two characteristics are interconnected in the sense that features of vulnerability coexist with features of resilience, with one or the other being more prominent depending on which layer of network hierarchy and which time horizon are considered. In view of this, the following discussion will consider resilience and vulnerability as features to be jointly assessed by distinguishing between different levels of network structure.

The distinction between interdependence and connectivity (see above) suggests a separation between resilience/vulnerability of structure and resilience/vulnerability of connections. They may coexist but they have different root causes, and they may lead to different outcomes. Resilience/vulnerability of structure is generated by the distribution of elements within a network, while resilience/ vulnerability of connections reflects the strengths of linkages between elements. ${ }^{13}$ For example, a network structure characterized by a flat hierarchy between elements is likely to be more vulnerable than a network structure characterized by a deep, multi-layered hierarchy of elements (Simon 1962). On the other hand, vulnerability of connections results from certain types of dominance of weak over strong linkages within the network (Simon 1962). The two types of vulnerability are related but do not coincide as they may be effective at different levels of structure or within different time periods. For example, a flathierarchy network may be vulnerable from the connectivity point of view even if its structure is relatively resilient to shocks. A case in point is that of a near-decomposable system characterized by dominance of weak ties between subsystems. In this case, the network is subject to vulnerability of connections in the sense that certain connections between subsystems are weak and may fall apart were the network subject to a significantly strong disturbance. At the same time, the network structure may be strong enough to withstand the disturbance precisely because of the low inter-system connectivity resulting from weak links between subsystems.

In short, vulnerability of connections could make network structure less vulnerable to shocks while vulnerability of structure could make the network more volatile, thus opening the way to new forms of connectivity and eventually to the emergence of new network structures. A near decomposable network exemplifies the coexistence of weak and strong ties and the implications of the latter for the relationship between

\footnotetext{
${ }^{13}$ This property may have significant implications for the vulnerability/resilience of connectivity in spatial networks characterized by the relative persistence of relative positions and by the changing strengths of linkages due to changing measures of relative distances (see Illenberger et al. 2013).
} 
vulnerability of connections and vulnerability of structure. As a matter of fact, a near decomposable network may be considered as a 'composite system [...] constructed by the superposition of: (1) terms representing interactions of the variables within each subsystem; and (2) terms representing interactions among the subsystems' (Simon and Ando 1961). In this type of system 'over a relatively short period [...] each subsystem can be' studied (approximately) independently of other subsystems. Over a relatively long period of time, on the other hand, [...] the whole system moves, keeping the state of equilibrium within each subsystem - i.e. the variables within each subsystem move roughly proportionately' (Simon and Ando 1961, p. 205).

This network structure shows that coexistence of weak and strong ties is likely to involve a combination of vulnerability and resilience: individual subsystems may be vulnerable while the whole system is resilient in the short period, but both individual subsystems and the whole system may be vulnerable to shocks if a sufficiently long time period is considered.

\subsection{Network Structure and Dynamic Impulses}

Different aggregation/decomposition criteria are associated with different combinations of weak and strong linkages between system components and are likely to involve different combinations of resilience and vulnerability for the network structure under consideration. As we have seen, aggregation/decomposition of activities into a system of industries highlights the interdependence of those activities into a potentially coherent and 'self-replacing' network of processes delivering intermediate products to one another. A given system of industries can show an internal hierarchy of activities depending on whether activities are delivering intermediate products to all remaining industries, or to some industries but not others, or to no other industry at all. This hierarchy has implications for the resilience/vulnerability of network structure seeing that a dynamic impulse (such as a technical innovation, or a resource bottleneck) is likely to trigger different responses depending on whether the industry directly affected occupies a central or a peripheral position in that hierarchy. The distinction between basic and non-basic industries is relevant in this connection (Sraffa 1960; Mathur 1966).

We may conjecture that a dynamic impulse directly affecting a basic industry is likely to trigger a more immediate and /or more significant effect than a dynamic impulse directly involving a non-basic industry. ${ }^{14}$ This means that a network of industries may be more vulnerable or less vulnerable to a negative shock depending

\footnotetext{
${ }^{14}$ As W.P. Strassmann noted 'induced technological changes in interrelated capital goods industries have been observed since the beginning of the industrial revolution. The first reciprocating steam engine built by James Watt was sold to John Wilkinson to power the bellows of Wilkinson's iron works at Brosely, England, and could itself be constructed only because Wilkinson had invented a new method of precision boring. A new method of metal-working thus led to power equipment which further increased productivity in the manufacture of metal goods. Later, the enormous generator rings and magnet frames of the world's first large hydroelectric power station at Niagara Falls could not be machined by conventional stationary machine tools. Portable tools had to be invented' (Strassmann 1959, p. 21). On the other hand, '[c]onsumer-oriented industries, such as textiles, participate only partially in these "feed-back loops" because the output of any particular stage of production does not often constitute the input of some preceding stage. Thread is not used in the construction of spinning machines; nor are the tools that make looms, or the engines that power them, made of cloth' (Strassmann 1959, p. 21).
} 
on the specific sub-system directly affected by the shock. A shock affecting a subsystem of basic industries is more likely to have a disruptive impact on the whole network relative to a shock affecting a peripheral subsystem (say, a non-basic industry or a set of non-basic industries). This means that a production network consisting of a core set of basic industries surrounded by a 'belt' of non-basic industries would be less vulnerable to negative shocks affecting its protective belt of non-basic industries provided the shocks directly affect the protective belt but not the core. On the other hand, the same network is more vulnerable to shocks affecting its core industries relative to a network characterized by a more polycentric arrangement of industries, as in the latter case negative shocks are likely to have effects contained within the neighbourhood of the affected activities without immediately spreading to the rest of the system.

A similar reasoning applies to the case of aggregation/decomposition by vertically integrated sectors. Here the production network (say, the collection of activities carried out in each district or country) may be associated with a collection of vertically integrated sectors $\mathbf{h}_{i} \mathrm{~s}: \boldsymbol{H}=\left[\mathbf{h}_{1}, \mathbf{h}_{2, \ldots,} \mathbf{h}_{\mathrm{m}}\right]$. As we have seen, not all vectors of intermediate product stocks need to belong to the same system of industries. This means that vulnerability and resilience, in this case too, will depend on which vertically integrated sector will be directly affected by a negative shock. In general terms, we may envisage situations in which individual vertically integrated sectors may be vulnerable to shocks specific to them while the whole system could be more resilient if enough vertical sectors (chains of production) belong to different systems of industries (see also Section 3).

Table 1 summarizes the relationship between vulnerability and resilience under the circular vs. vertical aggregation/decomposition of economic activities.

Table 1 shows different distributions of vulnerability/resilience as we move from horizontal to vertical aggregation/decomposition or vice versa. With horizontal aggregation/decomposition around a single core of basic industries, resilience would be maximized in the case of impulses affecting peripheral industries while vulnerability would be maximized in the case of impulses directly affecting the core of the network of industries under consideration (see also Reggiani et al. 2002; Modica and Reggiani 2015). On the other hand, in the case of the aggregation of activities into a collection of distinct vertically integrated sectors (chains of production), resilience would be maximized in the case of impulses separately affecting one or more disjointed sectors but leaving the other sectors unaffected, while vulnerability would be maximized in the case of impulses disrupting some vertical sectors and spreading to other sectors through the channels provided by the relevant network of industries (this network may be

Table 1 Network structure, decomposition, and resilience /vulnerability

\begin{tabular}{lll}
\hline & Resilience & Vulnerability \\
\hline $\begin{array}{ll}\text { Horizontal decomposition } \\
\text { Vertical decomposition }\end{array}$ & $\begin{array}{l}\text { Peripheral industries affected } \\
\text { Disjointed sectors affected }\end{array}$ & $\begin{array}{l}\text { Single core of basic industries affected } \\
\text { Connected sectors affected }\end{array}$ \\
\hline
\end{tabular}


contained within a single region or country or may be cutting across the borders of different regions or countries). In other words, the horizontal vs. vertical distinction calls attention to a major distinction between different sources of vulnerability and resilience.

The horizontal approach gives prominence to systems of activities that are mutually necessary, so that its emphasis would be on the inter-industry network within the economy (the subsystem of production processes delivering intermediate products to one another) (Leontief 1991 [1928], 1941). This approach looks at network structure from what we may call a 'centric' point of view since both vulnerability and resilience reflect whether impulses have direct influence on the inter-industry core or on peripheral subsystems. Activities belonging to the core make network structure vulnerable, in the sense that the operation of the whole network may cease or be seriously hampered when activities in the core cease to operate. On the other hand, the very distinction between core and peripheral activities highlights resilience features of network structure since impulses affecting only peripheral activities are likely to leave that structure in existence even if its mode of operation may be changed.

Differently from the horizontal approach, the vertical approach highlights sequences of activities that may be carried out independently from one another. The emphasis of this approach is on parallel sequences of fabrication stages (chains of production) leading to the production of final goods (consumption or investment goods) under conditions of a stationary or growing economy (Pasinetti 1980 [1973], 1988). The vertical approach looks at network structure from what we may call a 'polycentric' point of view since both vulnerability and resilience reflect whether impulses have direct influence on vertically integrated sectors $\mathbf{h}_{i} s$ as well as whether they have some influence on the networks of industries providing the commodity stocks entering the vertical sectors $\mathbf{h}_{i} s$. A network structure consisting of several $\mathbf{h}_{i} s$ sectors running in parallel may be resilient to impulses disrupting only some of those sectors. On the other hand, the $\mathbf{h}_{i} s$ sectors may receive intermediate commodity stocks from different 'horizontal' networks of industries, so that an impulse compatible with the resilience of the matrix of vertically integrated sectors $\boldsymbol{H}=\left[\mathbf{h}_{1}\right.$, $\left.\mathbf{h}_{2, \ldots,}, \mathbf{h}_{\mathrm{m}}\right]$ may have a disruptive effect on one or another of the networks of industries that provide the commodity stocks entering matrix $\boldsymbol{H}$. The latter feature highlights that resilience shown by one aggregation/decomposition criterion may be compatible with vulnerability shown by a different aggregation/decomposition criterion. This property calls attention to the context dependence of resilience/vulnerability and to the need of identifying what is the dominant aggregation/decomposition criterion in each case.

\section{Architecture of Complexity and Patterns of Interdependence: Towards a Compositional Approach to Structural Dynamics and Policy}

\subsection{Structural Economic Dynamics: Horizontal Structures and Scale-Free Networks}

Structural economic dynamics may be defined in a plurality of ways (see, for example, Silva and Teixeira (2008). A common feature of its definitions is emphasis on the positional characteristics of system elements. This means that system elements are identified primarily in terms of their relative positions with respect to other elements within a given network. In this light, structural dynamics involves the change of relative positions of network elements 
with respect one another (Scazzieri 2012, 2017; Hagemann et al. 2003; Cardinale and Scazzieri 2019). It follows that a prevailing mode of aggregation /decomposition, by determining the distribution and hierarchy of relative invariances in the system, may be a critical factor in determining which positional changes are compatible with the permanence of the given network structure. As a result, prevailing modes of aggregation/decomposition may explain prevailing modes of positional variation between system elements and therefore also prevailing trajectories of structural dynamics. For example, dominance of the horizontal mode of decomposition makes an economic and spatial network resilient to dynamic impulses affecting peripheral industries but vulnerable to impulses directly affecting core industries (industries delivering intermediate inputs to one another and to all other industries in the system). This means that certain impulses (say, a resource bottleneck or a fall in demand for certain final products) may leave relative positions within the core set of industries unaffected while inducing a change in the relative positions of peripheral industries. In this case, a horizontally structured economic system may show a degree of resilience not to be found in a system of vertically integrated sectors, provided the core set of industries is able to support manifold combinations of final consumption or investment goods.

On the other hand, an impulse affecting one or more basic industries is likely to induce a change of relative positions within the core set of industries, and is therefore likely to trigger a more radical type of structural change (the switch from one set of basic industries to another). This property of hierarchically ordered horizontal structures is consistent with the property of scale-free networks by which 'the probability that a node is highly connected is statistically more significant than in a random graph, the network's properties often being determined by a relatively small number of highly connected nodes that are known as hubs' (Barabási and Oltvai 2004, p. 105; see also Barabási and Albert 1999; Boccaletti et al. 2006). As with the hierarchically ordered horizontal structures discussed here, scale-free networks would be more vulnerable to shocks affecting the hubs, although they are more likely to be resilient to random shocks, which would likely to be distributed between hubs and other elements of the network (in our case, between 'peripheral' and 'core' industries) (see Barabási and Oltvai 2004, p. 110).

\subsection{Structural Economic Dynamics: Vertical Structures and the Context-dependence of Transformation}

If we move to networks characterized by dominance of decomposition into vertically integrated sectors, the same type of impulse would trigger consequences different from the horizontal case. For that impulse may or may not lead to significant changes in the relative proportions between different sectors in matrix $\boldsymbol{H}=\left[\mathbf{h}_{1}, \mathbf{h}_{2, \ldots,} \mathbf{h}_{\mathrm{m}}\right]$ depending on the commodity stock composition of vectors $\mathbf{h}_{i} s$. We may envisage situations in which the impulse would not significantly affect the relative proportions between vectors $\mathbf{h}_{i} s$ and other situations in which the same impulse would induce significant changes in the relative proportions between those vectors. For example, a fall in the demand for the final consumption good produced by vertically integrated sector $\mathbf{h}_{\mathrm{k}}$ may or may not bring about the deindustrialization of the whole economic network supporting $\mathbf{h}_{\mathrm{k}}$ depending on the size of $\mathbf{h}_{\mathrm{k}}$ relative to the other vertically integrated sectors in matrix $\boldsymbol{H}$. An economic and spatial network with manufacturing production too heavily concentrated in a single vertically integrated sector is more likely to fall back to a 
pre-industrial configuration of production activities than networks endowed with more versatile core industries ${ }^{15}$ In addition, there could be cases in which minor changes in the relative proportions between the vertically integrated sectors entering matrix $\boldsymbol{H}$ involve a major transformation in one or more of the networks of industries providing the commodity stocks entering vectors $\mathbf{h}_{i}$ s. $^{16}$

\subsection{Policy, Unintended Outcomes, and the Relevance of Theory}

The above argument is especially relevant from the policy point of view. A given policy action may be considered as an impulse aimed at triggering a certain outcome, be it in the short-, medium-, or long-term. Policy may or may not be explicitly aimed at inducing structural change. However, it may in any case trigger a structural dynamics trajectory, independently of whether it is aimed to do so or not. Indeed, certain policy actions may induce a system reconfiguration with significant, and not necessarily desired, unintended outcomes. For example, innovation policy inducing radical innovation in one or more basic industries may trigger the switch from one set of basic industries to another, which may in turn 'destabilize' the overall system in the sense that relatively stable intermediate forms (in Simon's sense) may be weakened or disappear, thereby reducing the sustainability of the very innovative potential that innovation policy aimed to enhance. ${ }^{17}$

In conclusion, dominance of either horizontal or vertical decomposition of network structure is key in explaining the type of structural change trajectory that dynamic impulses may induce in each context. The dominance of one or another decomposition criterion has important consequences both for theoretical and empirical research.

Theory is a necessary condition for context-relevant empirical work as it provides the heuristic needed for detecting which linkages generate the fundamental architecture of network structure (whether these linkages are of the horizontal or of the vertical type).

Empirical investigation informed by theory is necessary for identifying which mode of decomposition is dominant in each context. Both theoretical and empirical research are necessary to identifying the dominant trajectory of structural change along which network structure is moving. In turn, this would be a prerequisite for assessing that trajectory against a desired benchmark, and to design a policy that would enhance that trajectory, or to make the economy to switch to a different one. ${ }^{18}$

\footnotetext{
15 John Hicks notes this point when discussing the case of Italy and Flanders in the sixteenth and seventeenth centuries: 'it is surely no accident that their early industrialisms, as compared with those of our day, were very narrowly based. They did indeed have other industries besides textiles; but the concentration upon textiles- the necessary concentration, in the technical conditions of that day- was such that, when their advantage in textiles was gone, there was nothing else to which they could turn' (Hicks 1959, p. 174)

16 These industrial networks may at least partly be external to the network structure (the economic system) covered by matrix $\mathbf{H}$ (see section 4 ).

${ }^{17} \mathrm{I}$ am grateful to an anonymous referee for pointing out the possibility of unintended policy outcomes and their impact on the resilience features of production networks.

${ }^{18}$ One important consequence of the openness of network structures to a variety of decomposition modes is that different modes of decomposition highlight different ways in which a particular shock may affect specific elements (say, specific industries) or the network structure as a whole. As we have seen, there may also be cases in which switching from one decomposition to another may have the effect of 'switching' specific elements (say, a specific industry) from one network structure to another. This property reinforces Cardinale's finding that vulnerability and resilience in view of systemic interest may not be associated with 'a univocally determined objective' (Cardinale 2019), seeing that even systemic interest may depend on what is the dominant decomposition in the network structure under consideration.
} 
Funding Open Access funding provided by Alma Mater Studiorum - Università di Bologna.

Open Access This article is licensed under a Creative Commons Attribution 4.0 International License, which permits use, sharing, adaptation, distribution and reproduction in any medium or format, as long as you give appropriate credit to the original author(s) and the source, provide a link to the Creative Commons licence, and indicate if changes were made. The images or other third party material in this article are included in the article's Creative Commons licence, unless indicated otherwise in a credit line to the material. If material is not included in the article's Creative Commons licence and your intended use is not permitted by statutory regulation or exceeds the permitted use, you will need to obtain permission directly from the copyright holder. To view a copy of this licence, visit http://creativecommons.org/licenses/by/4.0/.

\section{References}

Adger WN (2006) Vulnerability. Glob Environ Chang 16:268-281. https://doi.org/10.1016/j.gloenvcha.2006. 02.006

Ames E, Rosenberg N (1965) The progressive division and specialization of industries. J Dev Stud 1(4):363383

Arthur WB, Landesmann M, Scazzieri R (1991) Dynamics and structures. Struct Chang Econ Dyn 2(1, June): $1-7$

Baas NA (1994) 'Emergence, hierarchies and Hyperstructures', Artificial Life III, Proceedings, ed. C.G. Langton, Addison-Wesley/Santa Fe Institute in the Sciences of complexity, vol xvii, pp 515-537

Barabási A-L, Albert R (1999) Emerging of scaling in random networks. Science 286:509-512

Barabási A-L, Oltvai ZN (2004) Networks biology: understanding the Cell's functional organisation. Nat Rev Genet 5:101-113. https://doi.org/10.1038/nrg1272

Beccaria C (1971 [ms. circa1769]) Elementi di economia pubblica, in C. Beccaria, Opere, a cura di S. Romagnoli, Firenze, Sansoni, vol I, pp 383-649

Bellino E (2018) Viability, reproducibility and returns in production price systems. Econ Polit 35:845-861

Boccaletti S, Latora V, Moreno Y, Chavez M, Hwang DU (2006) Complex networks: structure and dynamics. Phys Reports 424(4-5):175-308

Bourdieu P (2012) Sur l'Etat. Cours au Collège de France, Paris, Raisons d'agir/Seuil

Bruneau M, Chang S, Eguchi R, Lee G, O’Rourke T, Reinhorn A, Shinozuka M, Tierney K, Wallace W, von Winterfeldt D (2003) A framework to quantitatively assess and enhance seismic resilience of communities. Earthquake Spectra 19:733-752

Cardinale I (2019) Vulnerability, resilience and 'systemic interest': a connectivity approach, Networks and Spatial Economics, April, pp 1-17, https://doi.org/10.1007/s11067-019-09462-9

Cardinale I, Scazzieri R (2019) Explaining structural change: actions and transformations. Struct Chang Econ Dyn 51:393-404

Dagum C (1969) Structural permanence: its role in the analysis of structural dualisms and dependences and for prediction and decision purposes. Zeitschrift für die gesamte Staatswissenschaft / J Instit Theoretic Econ 125(2):211-235

Dahmén E (1970) Entrepreneurial activity and the development of Swedish industry, 1919-1939; translated by Axel Leijonhufvud, Homewood, R. D. Irwin

Dahmén E (1984) Schumpeterian dynamics: some methodological notes. J Econ Behav Organiz 5:25-34

Ducruet C, Beauguitte L (2014) Spatial science and network science: review and outcomes of a complex relationship. Netw Spat Econ 14:297-316

Fligstein N, McAdam D (2011) Toward a general theory of strategic action fields. Sociol Theory 29(1):1-26 Fligstein N, McAdam D (2012) A theory of fields. Oxford University Press, Oxford

Forsyth AR (1893) Theory of functions of a complex variable. Cambridge University Press, Cambridge

Goyal S (2007) Connections: an introduction to the economics of networks. Princeton University Press, Princeton

Hagemann H, Landesmann MA, Scazzieri R (2003) Introduction. In: Hagemann H, Landesmann M, Scazzieri $\mathrm{R}$ (eds) The economics of structural change, vol I. International Library of Critical Writings in Economics, Cheltenham, and Northampton, Massachusetts, Edward Elgar, Cheltenham, UK and Nortampton, Masssachutess, pp 11-43

Hicks JR (1959) Essays in world economics. Clarendon Press, Oxford 
Hicks JR (1977 [1973]) The mainspring of economic growth. In: Hicks J (ed) Economic perspectives. Further Essays on Money and Growth. Clarendon Press, Oxford, pp 1-19

Holling CS (1973) Resilience and stability of ecological systems. Annual Rev Ecol Syst 4:1-23

Illenberger J, Nagel K, Flotterod G (2013) The role of spatial interaction in social networks. Netw Spat Econ 1:255-282

Landesmann MA, Scazzieri R (1990) Specification of structure and economic dynamics. In: Baranzini M, Scazzieri R (eds) The economic theory of structure and change. Cambridge University Press, Cambridge, pp 95-121

Landesmann MA, Scazzieri R (1996) Coordination of production processes, subsystem dynamics and structural change. In: Landesmann MA, Scazzieri R (eds) Production and economic dynamics. Cambridge University Press, Cambridge, pp 304-343

Leontief W (1928) Die Wirtschaft als Kreislauf. Archiv für Sozialwissenschaft und Sozialpolitik 60:577-623

Leontief W (1941) The structure of the American economy. Oxford University Press, New York

Leontief W (1991 [1928]) The economy as circular flow. Struct Chang Econ Dyn 2(1, June):181-212

Leontief W et al (1953) Studies in the structure of the American economy; theoretical and empirical explorations in input-output. Oxford University Press, New York

Mathur G (1966) Planning for steady growth. Basil Blackwell, Oxford

Modica M, Reggiani A (2015) Spatial economic resilience: overview and perspectives. Netw Spat Econ 1: 211-233

Naimpally SA, Warrack BD (1970) Proximity spaces. Cambridge University Press, Cambridge

Pasinetti LL (1977) Lectures on the theory of production. Columbia University Press, New York

Pasinetti LL (1980 [1973]) The notion of vertical integration in economic analysis. In: Pasinetti LL (ed) Essays on the theory of joint production. London and Basingstoke, Macmillan, pp 16-43

Pasinetti LL (1988) Growing sub-systems, vertically hyper-integrated sectors and the labour theory of value. Camb J Econ 12:125-134

Piaget J (1971 [1968]) Structuralism. Routledge and Kegan Paul, London

Quadrio Curzio A (1986) Technological scarcity: an essay on production and structural change. In: Baranzini M, Scazzieri R (eds) Foundations of Economics. Structures of Inquiry and Economic Theory. Basil Blackwell, Oxford, pp 311-338

Quadrio Curzio A. (1967) Rendita e distribuzione in un modello economico plurisettoriale, Milano, Giuffrè

Quadrio Curzio A (1996) Production and efficiency with global technologies. In: Landesmann M, Scazzieri R (eds) Production and economic dynamics. Cambridge University Press, Cambridge, pp 105-126

Quadrio Curzio A, Pellizzari F (1999) Rent, Resources, Technologies. Springer, Berlin, Heidelberg, New York, etc.

Quesnay F (1972 [1759]) Quesnay s Tableau économique, edited, with new material, translations and notes by M. Kuczynski and R. L. Meek, London, Macmillan and New York, A. M. Kelley, for the Royal Economic Society and the American economic association

Reggiani A (2013) Network resilience for transport security: some methodological considerations. Transp Policy 28:63-68

Reggiani A (2014) Complexity and spatial networks. In: Fischer MM, Nijkamp P (eds) Handbook of regional science. Springer-Verlag, Berlin and Heidelberg, pp 811-832

Reggiani A, De Graaff T, Nijkamp P (2002) Resilience: an evolutionary approach to spatial economic systems. Netw Spat Econ 2:211-229

Ricardo, D. (1951 [1817]) On the Principles of Political Economy and Taxation, in Sraffa, P., with the collaboration of M.H. Dobb (eds.), The Works and Correspondence of David Ricardo, vol. I, Cambridge, Cambridge University Press for the Royal Economic Society

Ross Ashby W (2004 [1962]) 'Principles of the self-organizing systems', Emergence: Complexity and Organization, special double issue, 6 (1-2, June), pp 102-126

Scazzieri R (1993) A Theory of Production. Tasks, Processes, and Technical Practices. Clarendon Press, Oxford

Scazzieri R (2012) Structural economic dynamics: methods, theories and decisions. In: Kurz H, Trautwein HM (eds) Essays in Honour of Harald Hagemann. Routledge, Abingdon, Oxfordshire (UK) and New York, pp 314-328

Scazzieri R (2014) A structural theory of increasing returns. Struct Chang Econ Dyn 29:75-88

Scazzieri R (2017) Liquidity architectures and production arrangements. In: Cardinale I, Coffman D, Scazzieri $\mathrm{R}$ (eds) The political economy of the Eurozone. Cambridge University Press, Cambridge, pp 155-182. https://doi.org/10.1017/9781316403730.008

Scazzieri R (2021) Complex Structures and Relative Invariance in Economic Dynamics, in Reggiani A, Schintler LA, Czamanski D, Patuelli R (eds) Handbook on Entropy, Complexity and Spatial Dynamics: A 
Rebirth of Theory?. Edward Elgar Publishing, Cheltenham, UK and Northampton MA, USA, forthcoming

Silva EG, Teixeira AAC (2008) Surveying Structural Change: Seminal Contributions and a Bibliometric Account. Struct Change Econ Dyn 19(4):273-300

Simon, HA (1962) 'Architecture of Complexity', Proceedings of the American Philosophical Society, vol. 106 (December), pp. 467-482.

Simon HA (1976) How complex are complex systems?, PSA: Proceedings of the Biennial Meeting of the Philosophy of Science Association, vol. two: Symposia and invited papers, pp 507-522

Simon HA, Ando A (1961) Aggregation of variables in dynamic systems. Econometrica 29(2, April):111-138

Sraffa P (1960) Production of commodities by means of commodities. Prelude to a critique of economic theory. Cambridge University Press, Cambridge

Strassmann WP (1959) Interrelated industries and the rate of technological change. Rev Econ Stud 27(1):1622

Tsiotas D, Polyzos S (2018) The complexity in the study of spatial networks: an epistemological approach. Netw Spat Econ 18:1-32

Yang X, Ng YK (1993) Specialization and economic organization. A New Classical Microeconomic Framework, Amsterdam-London-New York-Tokyo, North Holland

Publisher's note Springer Nature remains neutral with regard to jurisdictional claims in published maps and institutional affiliations.

\section{Affiliations}

\section{Roberto Scazzieri ${ }^{1,2}$}

1 Department of Economics, University of Bologna, Piazza Scaravilli, 2, 40126 Bologna, Italy

2 National Lincei Academy, Via della Lungara 10, 00165 Rome, Italy 